\title{
Ground Penetrating Radar (GPR): an application for evaluating the state of maintenance of the building coating
}

\author{
Giovanni Leucci, Sergio Negri and Maria Teresa Carrozzo \\ Osservatorio di Chimica, Fisica e Geologia Ambientali, \\ Dipartimento di Scienza dei Materiali, Università di Lecce, Italy
}

\begin{abstract}
Ground Penetrating Radar (GPR) is a non-destructive methodology. For the localisation of buried structures, it uses short time duration electromagnetic (EM) pulses lasting from about $1 \mathrm{~ns}$ to about $30 \mathrm{~ns}$. Therefore, GPR is characterised by a wide frequency band ranging from $10 \mathrm{MHz}$ to some $\mathrm{GHz}$, and is useful in the localisation of EM discontinuities in the subsurface with high resolution. This paper describes an application of GPR to evaluate the state of maintenance of some travertine panels attached to the inside walls of a building housing the Bank of Naples in Campobasso and in danger of falling because of the numerous voids present between the wall and the panels. The aim of the survey was to assess whether the GPR technique could be used to detect the voids behind the travertine panels. The study was made to assist the design of the restoration works of the travertine covering. Because of the very narrow thickness of both the travertine plates and the voids, special care was needed in the acquisition and processing steps. The measurements were performed on two panels: one purposely put on to the laboratory wall with a known position of the voids; the other one selected from among the panels to be restored. Although pushed to the limit of the resolution achievable by the available antenna, the study has given quite good results.
\end{abstract}

Key words GPR investigation - GPR resolutiontravertine panel-time slices

\section{Introduction}

The GPR method can detect superficial bodies with relative efficiency depending on the field context, the dielectric properties of the host material and the nature and size of the bodies (Davis and Annan, 1989). The scale of resolvable features can range from a few centimeters to ten or more meters, depending on the frequency band

Mailing address: Dr. Giovanni Leucci, Osservatorio di Chimica, Fisica e Geologia Ambientali, Dipartimento di Scienza dei Materiali, Università di Lecce, Via per Arnesano, 73100 Lecce, Italy; e-mail: gianni.leucci@unile.it of the operating antennas. Therefore, a careful selection among the available antennas is needed with regard to the aims of the survey (Annan and Cosway, 1994). Numerous studies describe efficient GPR techniques in civil engineering (Mellet, 1995; Bae et al., 1996; Carcione, 1996; Davidson and Chase, 1998; Godio et al., 1999; Leucci et al., 2002). However, since each technique is generally considered individually in a specific context, it is difficult to compare the results because of different field conditions.

The objective of this work is to verify the capabilities of GPR to estimate the state of maintenance of some travertine panels attached to the inside wall of the Bank of Naples in Campobasso.

These panels are affected by instability mainly caused by numerous voids, that denote an absence of mortar. The main target of these pre- 
liminary measures is therefore to verify the reliability of the GPR methodology to detect voids between the wall and the travertine panels. The measures were carried out on two panels:

- one in the laboratory with a known position and thickness of the voids;

- the second panel was placed inside the Bank of Naples in Campobasso, with the position and thickness of the voids completely unknown.

Because of the very narrow thickness of the travertine (less than $4 \mathrm{~cm}$ ), special care in the acquisition of the data on the voids (parameters of acquisition, geometry of acquisition) was required. Although the only available choice of antenna with the higher centre frequency band was $1 \mathrm{GHz}$ and therefore with well-known limits of resolution, the results seem to be satisfactory.

\section{Outline of the problem}

The Bank of Naples in Campobasso is located inside a building of ancient construction. The inside of the building is entirely covered with travertine panels $70 \mathrm{~cm}$ wide, $90 \mathrm{~cm}$ high and $3 \mathrm{~cm}$ thick. Last year the static condition of one of these panels presented some problems due to the presence of voids and therefore to the lack of mortar between the wall and the panel itself. Figure 1 represents a hypothetical stratigraphical section that shows the problem.

The problem (given the narrow thickness to investigate) is to establish whether GPR is able to map the void present between the wall and the travertine panel. The void detection is mainly based on the different EM properties between the travertine, the mortar, the wall and the air filling the voids. The size and the geometric shape of

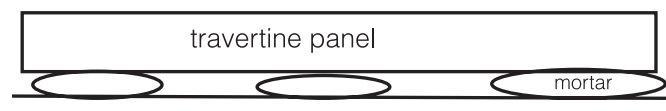

wall

Fig. 1. Hypothetical stratigraphical section with the panel of travertine $3 \mathrm{~cm}$ thick, the mortar with layer ranging from about $1 \mathrm{~cm}$ to some $\mathrm{cm}$, and the wall. the structures play an important role. In the range of the radar frequencies the EM wave velocity $v$ and the EM wave attenuation $\alpha$ in the medium are used to characterise the medium itself. In materials of low magnetic susceptibility, velocity $v$ and attenuation $\alpha$ are related to the Relative Dielectric Permettivity (RDP) $k$ and loss tangent, $\operatorname{tg} \delta$, by the expressions (Annan and Davis, 1988)

$$
\begin{gathered}
v=\frac{c}{\sqrt{k}} \\
\alpha=\frac{\omega \cdot \sqrt{k}}{2 \cdot c} \cdot \operatorname{tg} \delta
\end{gathered}
$$

where $\omega=2 \pi f$ is the angular frequency, $f$ is the frequency and $c$ is the velocity of the EM wave in the empty space $(30 \mathrm{~cm} / \mathrm{ns})$. The attenuation decreases as the frequency decreases (Davis and Annan, 1989). On this basis, the attenuation and, therefore the frequency play an important role in the penetration depth and both vertical and horizontal resolution for the GPR system. Vertical resolution is a measure of the ability to differentiate between two signals adjacent to each other in time. Simplistically, vertical resolution is a function of frequency. Each radar antenna is designed to operate over a range of frequencies (bandwidth) where the peak power occurs at the centre frequency of the antenna. The centre frequency is also inversely proportional to the pulse period (in nanoseconds). The equivalent length (in meters) of the pulse is the product of the pulse period and the EM wave velocity in the medium. Resolution can be taken as one-quarter of the wavelength $(\lambda)$ of EM wave (Burger, 1992)

$$
\lambda=v / f
$$

where $v$ is the EM wave velocity in the medium and $f$ is its frequency.

The horizontal resolution is related to the EM wave which travels from the transmitter antenna in a cone of radiation with a finite-sized footprint (fig. 2), (Conyers and Goodman, 1997). The first Fresnel zone describes the minimum area in which features with smaller dimensions will not 


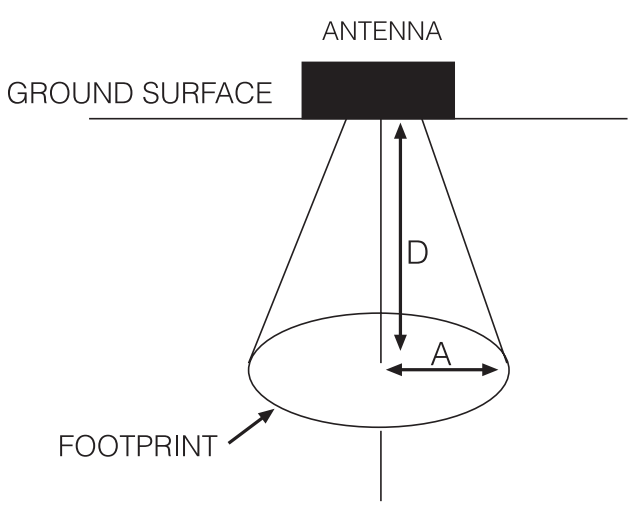

Fig. 2. The elliptical cone of GPR into the ground (from Conyers and Goodman, 1997).

be imaged. The finite size of this footprint affects both the vertical resolution (when interfaces are steeply dipping or have high amplitude surface roughness relative to the wavelength of the incident EM waves), and the horizontal resolution. The larger the first Fresnel zone, the lower the horizontal resolution in discriminating between adjacent targets will be. The radius of footprint is (Conyers and Goodman, 1997)

$$
A=\frac{\lambda}{4}+\frac{D}{\sqrt{K+1}}
$$

where $\lambda$ is the center frequency wavelength of
EM wave, $D$ is the depth from the ground surface to the reflection surface, $k$ is the relative dielectric permettivity of material from the ground to the surface depth $(D)$.

Horizontal resolution is inversely proportional to $(\alpha)^{1 / 2}$, (Burger, 1992). Consequently, the horizontal resolution is actually better over a high-loss material than over a low-loss medium. The analysis directly performed on the radar sections (see next Section) acquired in a controlled situation and using the relationships (2.1), (2.3) and (2.4), allowed table I to be realized.

Table I summarises the problem well.

Another problem is the near-field effect. An EM wave radiated from a surface antenna generates an EM field around the antenna within a radius of about 1.5 wavelengths of the center frequency (Balanis, 1989; Sheriff, 1984). For the $1 \mathrm{GHz}$ antenna this effect is approximately $30 \mathrm{~cm}$ if the EM wave radiated in air (Conyers and Goodman, 1997), while it is approximately $17 \mathrm{~cm}$ if the EM wave radiated in travertine. Within this zone, the EM wave is coupled with the ground, generating an advancing wave front in the standard conical transmission pattern outside the radius. It can be said that the ground within about 1.5 wavelengths of a standard dipole antenna is part of the antenna where no radiation is occurring within this zone, and therefore technically no propagation. In the GPR sections, this zone is visible as an area of few or no reflections. To obviate the near-field effect zone a travertine layer $(6 \mathrm{~cm}$ thick) between the antenna and the panel was used in the survey.

Table I. The travertine, mortar and empty space (air) EM properties with vertical resolution $\lambda / 4$ and footprint radius $A$.

\begin{tabular}{ccccc}
\hline \hline $1 \mathrm{GHz}$ antenna, $D=10 \mathrm{~cm}$ & \multicolumn{3}{c}{} \\
\hline Material & Velocity $(\mathrm{cm} / \mathrm{ns})$ & $k$ & $\lambda / 4(\mathrm{~cm})$ & $A(\mathrm{~cm})$ \\
\hline Travetine & 11.4 & 6.9 & 2.85 & 6.4 \\
Mortar & 9.2 & 10.7 & 2.3 & 5.2 \\
Travertine + air & 12.8 & 5.5 & 3.2 & 7.1 \\
Travertine + mortar & 11 & 6 & 2.75 & 6.5 \\
Air & 30 & 1 & 7.5 & 14.6 \\
\hline
\end{tabular}




\section{Field instrumentation, data acquisition and analysis}

The GPR survey was carried out with a georadar System-2 (SIR 2) and a $1 \mathrm{GHz}$ (centre frequency) antenna manufactured by Geophysical Survey Systems Inc. (GSSI). The $1 \mathrm{GHz}$ antenna selection was due to the resolution required by the survey objectives.

The following acquisition parameters were selected:

- data word length: 8 bit;

- samples per scan: 512;

- recording time window: $10 \mathrm{~ns}$;

- gain function: manual (5 gain points $(12,12$, $22,35,47) \mathrm{dB}$ );

- reference marks distance: $10 \mathrm{~cm}$.

To obviate the near-field effect zone, a travertine layer ( $6 \mathrm{~cm}$ thick) between the antenna and the panel was used in the survey.

\subsection{Measures in the test site (travertine panel hung in the laboratory)}

A travertine panel $(100 \mathrm{~cm}$ wide, $80 \mathrm{~cm}$ high and $3 \mathrm{~cm}$ thick) was hung in the laboratory (fig. $3 a, b)$. A layer of mortar was hung between the wall and the panel $(20 \mathrm{~cm}$ wide, $80 \mathrm{~cm}$ high and $2 \mathrm{~cm}$ thick) every $20 \mathrm{~cm}$ on the whole length of the panel. Such $20 \mathrm{~cm}$ of mortar and $20 \mathrm{~cm}$ of void were alternated along the whole panel (fig. 3a). A reconnaissance survey was made in continuous mode, in a rectangular area of $100 \mathrm{~cm}$ by $80 \mathrm{~cm}$, along $5 \mathrm{~cm}$ spaced parallel profiles. The problem with the data acquisition is the near field.

The quality of the original data did not require advanced processing techniques. In fact, only horizontal scaling normalisation $(2 \mathrm{scan} / \mathrm{cm})$ was performed for an easier interpretation.

Figure 4 shows the reflection profile, labelled A2 in fig. 3a. An analysis of fig. 4 points out:

- The clear enough interface that represents the contact between the antenna and the interposed panel.

- The series of anomalies with the first arrivals between 1.7 and $2 \mathrm{~ns}$ that represent, given their position along the profile, alternatively the wallmortar interface $(2 \cdot n s)$ and wall-empty interface
(1.7 ns), the latter with clearly greater amplitude than those related to the wall-mortar interfaces. As already stated, we could not resolve the top and bottom of the mortar and voids. Table I used both the first arrivals related to wall-mortar interface $(2 \mathrm{~ns})$ and wall-empty interface $(1.7 \mathrm{~ns})$ and the relationship $v=(2 \cdot s) / t$ where $v$ is the EM wave

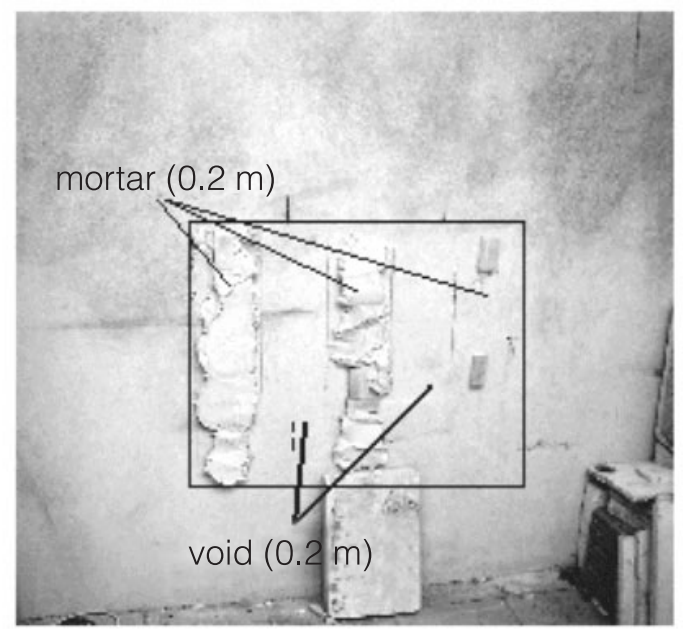

(a)

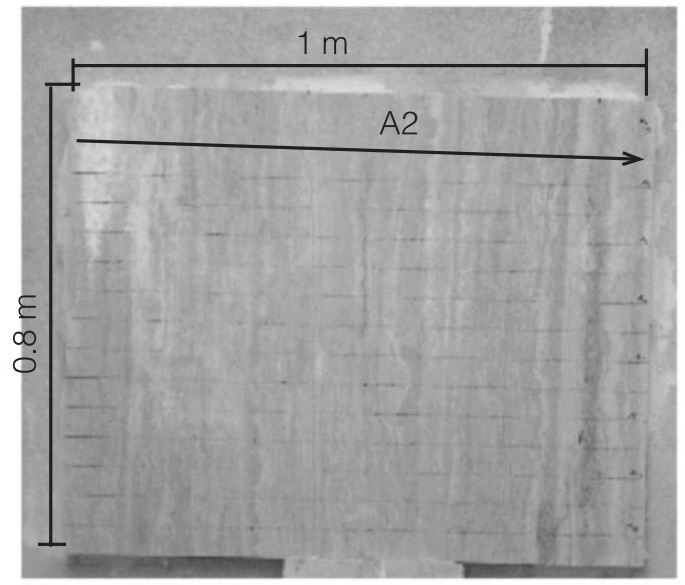

(b)

Fig. 3a,b. Photo of the travertine panel hung in the laboratory: a) before the assemblage on the wall with the position of mortar and void; b) hung on the wall with location of radar profile labelled A2. 


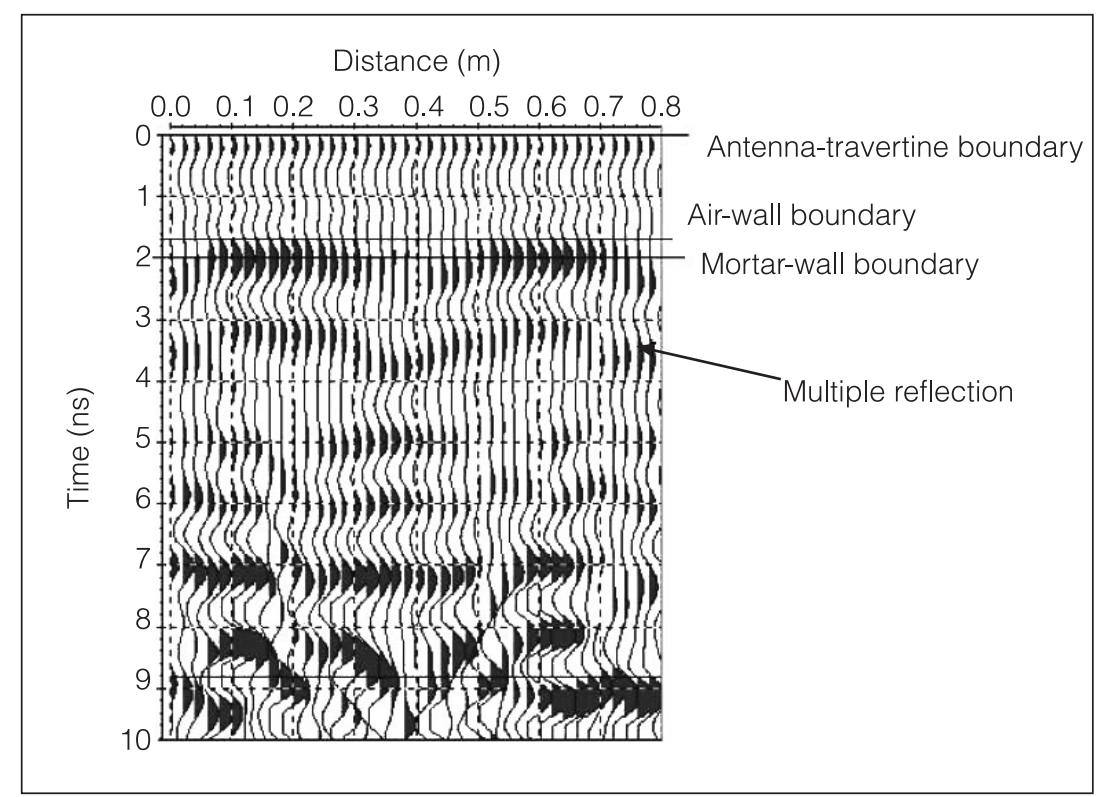

Fig. 4. Radar section related to the profile labelled A2 in fig. $3 b$.

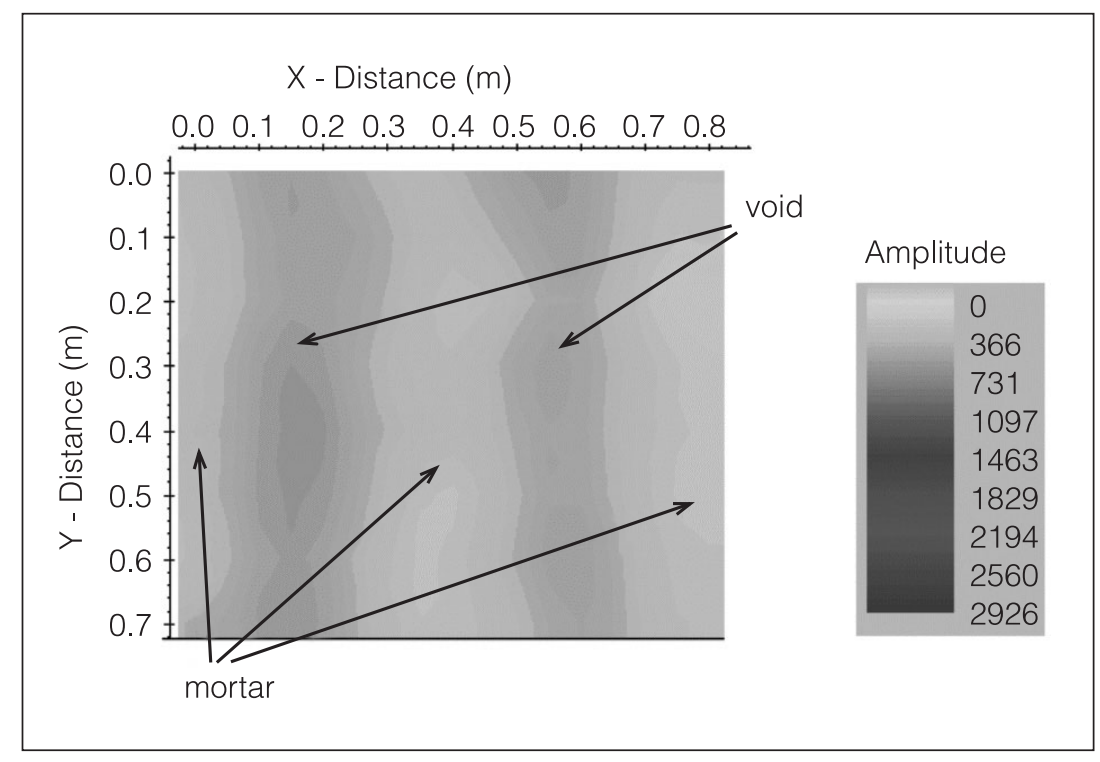

Fig. 5. Time slice for the $1 \mathrm{~ns}-3 \mathrm{~ns}$ time window with the energy amplitude values. Several events are visible as high amplitude anomalies (dark region) that coincide with the shape and position of the voids between the travertine panel and the wall. The weak amplitude anomalies (clear region) coincide with the shape and position of the mortar between the travertine panel and the wall. 
velocity (unknown) in the studied medium, $s$ is the measured depth to the voids and mortar (known) and $t$ is the two-way travel time from the surface to the voids and mortar and back to the surface.

One way to obtain visually useful maps to understand the plan distribution of reflection amplitudes within specific time intervals is the creation of horizontal time slices (Conyers and Goodman, 1997). This data representation plays an important role in GPR investigations as it allows an easier correlation of the most important anomalies found in the area at the same depth, thus facilitating the interpretation (Basile et al., 2000). In reality, because of possible velocity changes across the area and with depth, horizontal time slices must be considered only as approximate depth slices. However, this is generally sufficient for most common applications. In the present work, the time slice technique was used to display the amplitude variations within the 1-3 ns time window, where the majority anomalies were observed. The selected two-way time interval corresponds to a soil layer, approximately $12 \mathrm{~cm}$ thick, located between 6 and $18 \mathrm{~cm}$ in depth, where the voids were lodged. In the slice shown in fig. 5, several events are visible as high amplitude anomalies (dark region) that coincide with the shape and position of the voids between the travertine panel and the wall.

The weak amplitude anomalies (clear region) coincide with the shape and position of the mortar between the travertine panel and the wall.

\subsection{Measures in situ (travertine panel inside the Bank of Naples in Campobasso)}

A travertine panel set into the lower part of the wall (fig. 6), inside the Bank of Naples in Campobasso, was considered.

The data were acquired using the same acquisition parameters and the same shrewdness fixed in the preceding case of the measure on the test site (Section 3.1).

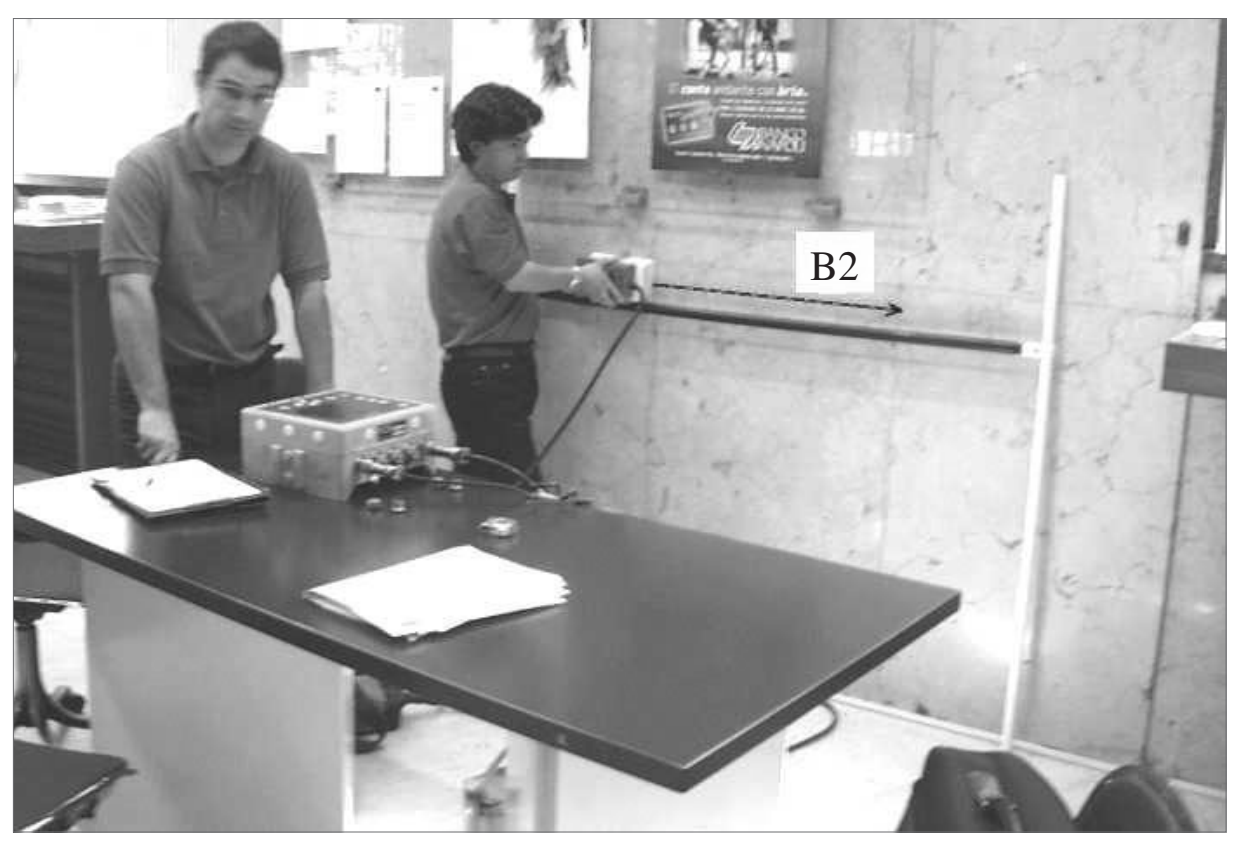

Fig. 6. Photo of the travertine panel inside to the Bank of Naples in Campobasso with the location of B2 radar profile. 


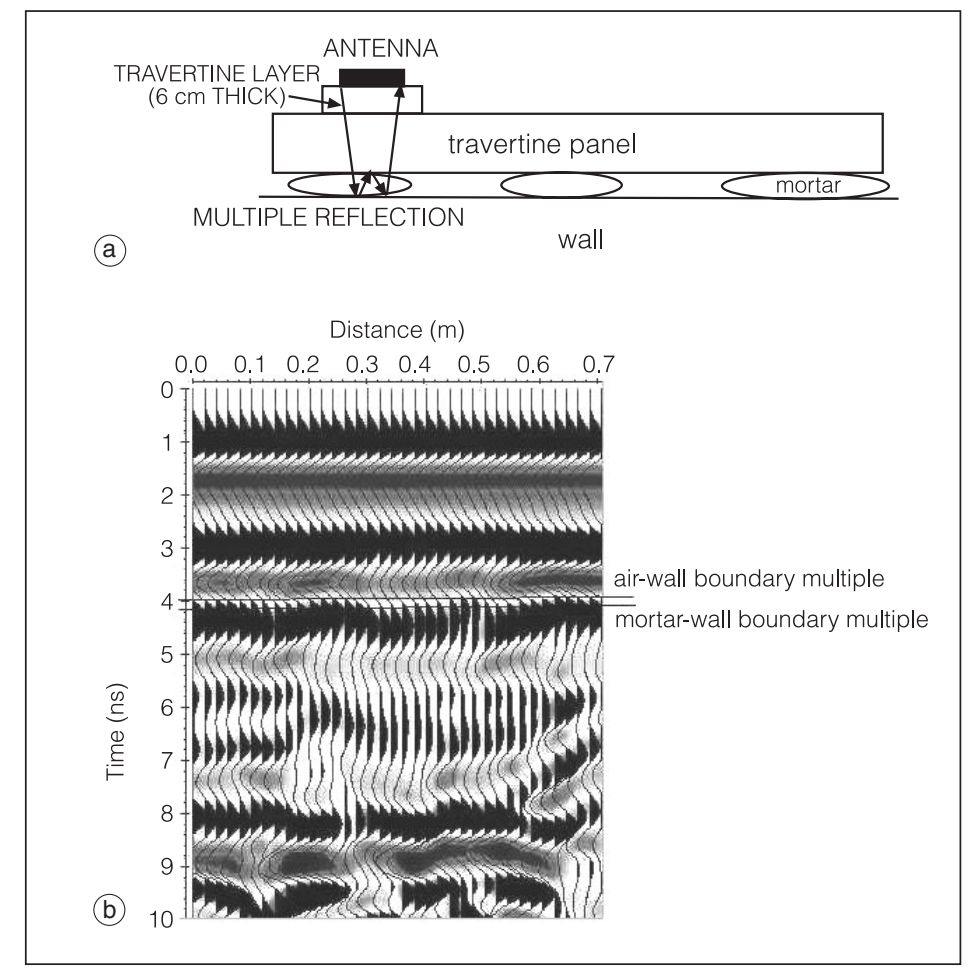

Fig. 7a,b. a) Model of radar transmission and reflection with multiple reflections off of subsurface interfaces prior to the wave's being recorded at the surface antenna; b) radar section related to the profile B2 shown in fig. 6 . The underlined multiple reflections are clearly related to the bottom of the interface air-wall and mortar-wall.

Figure 7 a shows the reflection profile (labelled B2) acquired in the upper part of the panel after the horizontal scaling normalisation $(2 \mathrm{scan} / \mathrm{cm})$ processing.

A general characteristic of all the profiles acquired on the panel is the near-field effect in the $0-3.4$ ns zone (fig. $7 \mathrm{a}$ ) corresponding to a depth from 0 to about $19 \mathrm{~cm}$ if the mean velocity value of $11.4 \mathrm{~cm} / \mathrm{ns}$ is used (see table I). Most of the expected anomalies are confined in this zone. The traditional background removal filter (Conyers and Goodman, 1997), applied to all data, does not allow the reflection events present in the near field zone to be emphasized. Usually, most of the EM waves that are reflected on a subsurface interface between two materials are transmitted directly back to the surface and recorded at the receiving antenna. Some of these reflected EM waves, however, can be re-reflected back into the subsurface at the ground surface-air interface, and then re-reflected back again on to the surface from the same buried interface prior to recording (fig. 7b). This multiple reflection effect creates a double echo of the subsurface reflector, with a recorded time about twice that measured for a wave reflected only once. The multiple phenomenon was also observed in the data acquired on the test site (fig. 4). In order to overcome the near field zone the multiple phenomenon was considered. Figure 7 a shows some interesting reflection events at about 4.2 and $4.0 \mathrm{~ns}$ (multiple reflections) that represent respectively the wall-mortar interface and wallempty interface. If the average value of the EM wave in the travertine + air is $12.8 \mathrm{~cm} / \mathrm{ns}$ (see table I) the void thickness is about $1 \mathrm{~cm}$. 


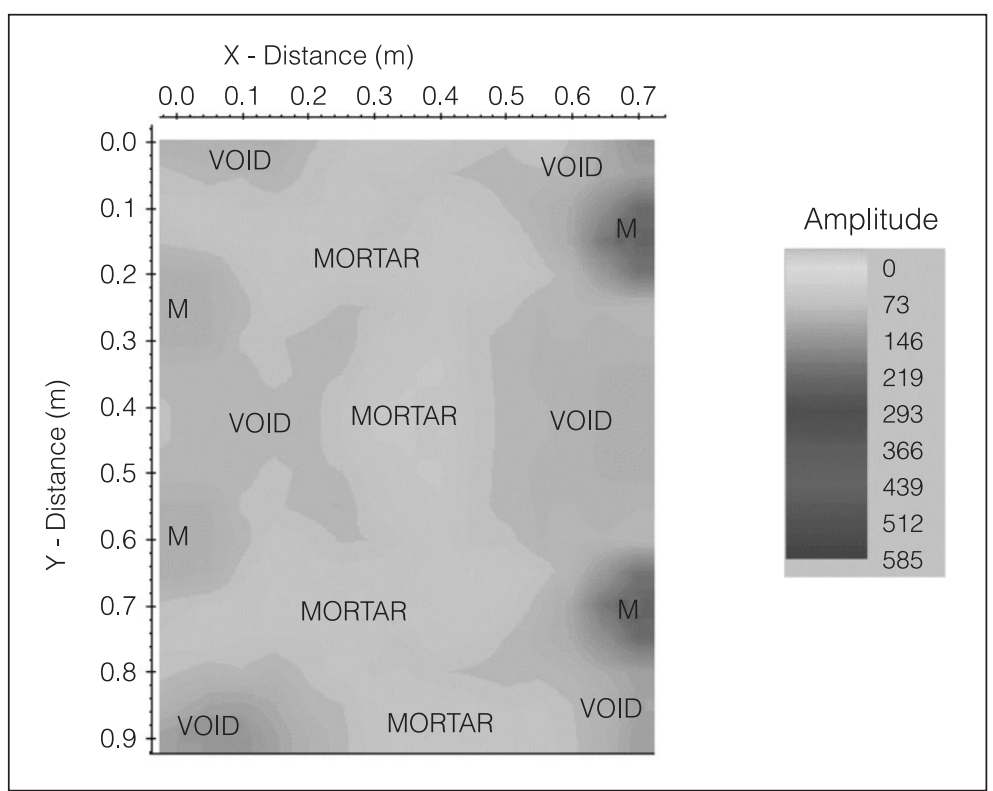

Fig. 8. Time slice for the $3.8 \mathrm{~ns}-5 \mathrm{~ns}$ time window with energy amplitude values. Note the presence of strong amplitude anomalies (labelled $M$ ) that, because of the shape and the position, could be correlated to metal objects placed to fix the panel. The anomalies of weaker amplitude, in comparison with those suitable with $M$ (labelled VOID), could be the voids.

A time slice map is also made in this case. The time interval from 3.8 to 5 ns was considered. The result is shown in fig. 8 .

The slice shows the presence of strong amplitude anomalies (labelled $M$ ) that, because of their shape and position, could be correlated to metal objects placed to fix the panel. The anomalies of weaker amplitude, in comparison with those suitable with $M$ (labelled VOID) could be the voids. Where the anomalies are more intense, the void thickness could be about $1 \mathrm{~cm}$. The clear zones in the slice (labelled Mortar) are probably related to the presence of the layer of mortar.

\section{Conclusions}

The GPR investigation was divided into two phases:

- the first one (testing phase) was carried out on a travertine panel hung in the laboratory with position and thickness of the voids known;
- the second one, considering the principal objective of the survey, was carried out on the panels of the travertine placed inside the Bank of Naples in Campobasso, with the position and thickness of the voids completely unknown.

The testing phases allowed a semiquantitative evaluation of the EM characteristic of the materials considered (table I) and disclosed more problems linked both to the methodology and geometry of the target.

The encouraging results obtained in the first phase of measures were used to understand and interpret the results obtained in the second phase of measures better. Using table I, and the hypothesis of similar electromagnetic conditions in the two phases, we have given a probable maximum thickness of void $(1 \mathrm{~cm})$ present between the travertine panel and the wall.

Even if they are still to be verified (to make a core boring on a panel the object of the investigation), the results obtained to date seem to be encouraging. 


\section{Acknowledgements}

The authors wish to thank the technician Serafino Corriero for his collaboration during data acquisition. The authors thank Dr. Tiziana di Biaso and Dr. Wendy Hudson for revising the English form of the manuscript.The authors are also grateful to the reviewers and to the Editor for their valuable comments.

\section{REFERENCES}

ANNAN, A.P. and J.L. DAVIS (1988): Radar sounding in potash mines, Saskatchewan, Canada, Geophysics, 53 (12), 1556-1564;

ANNAN, A.P. and S.W. CoswAY (1994): GPR frequency selection, in Proceedings of the Fifth International Conference on Ground Penetrating Radar, Waterloo Centre for Groundwater Research, Waterloo, Canada, 747-760.

BAE, S.H., H.S. KIM and W.S. YoON (1996): Cases studies on the application of ground penetrating radar technology in detection of underground utilities an structure safety diagnosis, in Proceedings of the Sixth International Conference on Ground Penetrating Radar, September 30-October 3, Sendai, Japan, 467-472.

BALANIS, C.A. (1989): Advanced Engineering Electromagnetics (John Wiley and Sons, New York), pp. 981.

BAsile, V., M.T. CARROZZO, S. NEGRI, L. NuZZO, T. QUARTA and A.V. VILLANI (2000): A ground-penetrating radar survey for archaeological investigations in urban area (Lecce, Italy), J. Appl. Geophys., 44, 15-32.

BURGER, H.R. (1992): Exploration Geophysics of the Shallow Subsurface; Englewood Cliffs, New Jersey, Print Hall, pp. 489.

CARCIONE, J.M. (1996): Ground-radar numerical modelling applied to engineering problems, Eur. J. Environ. Eng. Geophys., 1 (1), 65-82.

CONYERS, L.B. and D. GOODMAN (1997): Ground-Penetrating Radar: an Introduction for Archaeologist, AltaMira Press, Walnut Creek, London and New Delhi, pp. 232.

DAVIDSON, N.C. and S.B. CHASE (1998): Radar systems for tomographic bridge deck inspection, in Proceedings of the 7th International Conference on Ground Penetrating Radar, May 27-30, Laurence KS, vol. 2, 749-751.

DAVIS, J.L. and A.P. ANNAN (1989): Ground penetrating radar for high-resolution mapping of soil and rock stratigraphy, Geophysics, 37, 531-551.

Godio, A., L. SAMBUELLI and V. Socco (1999): Misure georadar per lo studio della volta della scarsella nel battistero del duomo di Firenze, in Proceedings 18th Convegno GNGTS, Roma 9-11 Novembre 1999, CDROM.

LEUCCI, G., S. NEGRI, M.T. CARROZZO and L. NUZZO (2002): Use of ground penetrating radar to map subsurface moisture variations in an urban area, J. Environ. Eng. Geophys., 7 (2), 69-77.

MELLET, J.S. (1995): Ground penetrating radar applications in engineering environmental management and geology, J. Appl. Geophys., 33, 157-166.

SHERIFF, R.E. (1984): Encyclopedic Dictionary of Exploration Geophysics, Second Edition, Society of Exploration Geophysics, Tulsa, Oklahoma, pp. 323. 